\title{
A Mixed Finite Element Method for Elasticity Problem
}

\author{
A. Elakkad \\ Department of mathematics \\ Regional Centre for Professions of Education and \\ Training, Fes, B.P: 243 Sefrou Morocco
}

\begin{abstract}
This paper describes a numerical solution for plane elasticity problem. It includes algorithms for discretization by mixed finite element methods. The discrete scheme allows the utilization of Brezzi - Douglas - Marini element $\left(B_{1} M_{1}\right)$ for the stress tensor and piecewise constant elements for the displacement. The numerical results are compared with some previously published works or with others coming from commercial code like ABAQUS.
\end{abstract}

keywords-Elasticity problem; Mixed Finite element method; $B D M_{1}$ approximation; $A B A Q U S$

\section{INTRODUCTION}

Mixed finite element methods for linear elasticity are based on approximations of a stress- displacement system derived from the Hellinger-Reissner variational principle [7], in which both displacements and stresses were approximated simultaneously.

The mathematical analysis and applications of mixed finite element methods have been widely developed since the seventies. A general analysis for this kind of methods was first developed by Brezzi [8]. We also have to mention the papers by Babuska [14] and by Crouzeix and Raviart [15] which, although for particular problems, introduced some of the fundamental ideas for the analysis of mixed methods.

We also refer the reader to [16][17], where general results were obtained, and to the books [6][18][19].

Many mixed finite element methods have been developed for plane elasticity, and generally speaking, they can be grouped into two categories: methods that enforce the symmetry of the stress weakly, and methods that enforce the symmetry exactly (strongly). In the former category, the stress tensor is not necessarily symmetric, but rather orthogonal to anti-symmetric tensors up to certain moments. Weakly imposed stress symmetry methods also introduce a new variable into the formulation that approximates the anti-symmetric part of the gradient of $\mathrm{u}$; see for example [2][3]. On other hand, exactly symmetric stress methods have been much more difficult to construct. The first class of

inf_sup stable methods was the so called composite elements [4][5].

\author{
M.A. Bennani, J. EL Mekkaoui and A.Elkhalfi \\ Mechanical engineering laboratory \\ Faculty of sciences and techniques-B.P. 2202 Route \\ Imouzzer Fes
}

Section II presents the model problem used in this paper. The discretization by mixed finite elements described is in section III. Numerical experiments carried out within the framework of this publication and their comparisons with other results are shown in section IV.

\section{GOVERNING EQUATIONS}

The equilibrium equations and boundary conditions are

$$
\begin{gathered}
\nabla . \sigma+f=0 \text { in } \Omega \\
\sigma . n=\bar{t} \text { on } \Gamma_{t} \\
\sigma . n=0 \text { on } \Gamma_{c+} \\
\sigma . n=0 \text { on } \Gamma_{c-}
\end{gathered}
$$

Where $\mathrm{n}$ is the unit outward normal. In the above, $\sigma$ is the Cauchy stress, and $\mathrm{f}$ is the body force per unit volume.

The constitutive relation is given by Hooke's law:

$$
\sigma=C: \varepsilon
$$

Where $\mathrm{C}$ is the Hooke tensor, $\mathrm{C}$ is assumed here to have constant coefficients. Its inverse (compliance tensor) will be denoted by E. Hence

$$
\sigma=C: \varepsilon \Leftrightarrow \varepsilon=E: \sigma .
$$

We consider small strains and displacements. The kinematics equations therefore consist of the straindisplacement relation

$$
\varepsilon=\varepsilon(u)=\nabla_{s} u
$$

$\varepsilon=\varepsilon(u)=\nabla_{S} u$
Where $\nabla_{s} u=\frac{1}{2}\left(\nabla u+\nabla u^{T}\right)$ is the symmetric part of the gradient operator, and the boundary condition

$$
u=\bar{u} \text { on } \Gamma_{u}
$$




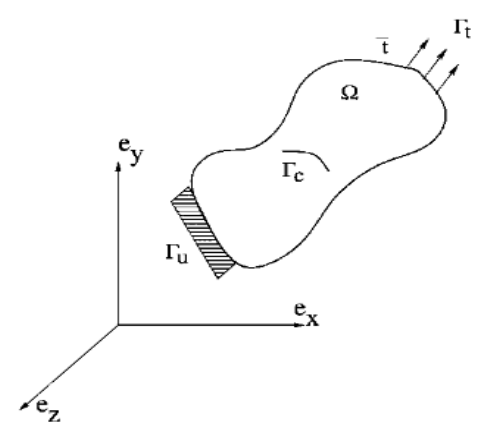

Fig. 1. Body With Internal Boundary Subjected To Loads.

We set

$$
\begin{gathered}
H(\operatorname{div}, \Omega)=\left\{\sigma \mid \sigma \in\left(L^{2}(\Omega)\right)^{4} ;\right. \\
\left.\sigma_{i j}=\sigma_{j i} \quad \forall i, j ; \operatorname{div} \sigma \in\left(L^{2}(\Omega)\right)^{2}\right\} . \\
H_{g}(\operatorname{div}, \Omega)=\left\{\tau \in H(\operatorname{div}, \Omega) ; \tau . n=g \text { on } \Gamma_{t}\right\} \\
\left.\in\left(L^{2}(\Omega)\right)^{2} ; u \text { discontinuous on } \Gamma_{c}\right\}
\end{gathered}
$$

Then the standard weak formulation of the equilibrium equations is the following:

Find $\sigma \in \mathrm{H}_{\bar{t}}(\operatorname{div}, \Omega)$ and $\mathrm{u} \in \mathrm{L}_{\text {dis }}^{2}(\Omega)$ such that:

$$
\begin{aligned}
& \int_{\Omega}(E: \sigma): \tau d x+\int_{\Omega} u \cdot d i v \tau d x \\
&= \int_{\Gamma_{u}} \bar{u} \tau \cdot n d \Gamma, \\
& \forall \tau \in H_{0}(d i v, \Omega) \\
& \int_{\Omega} v \cdot d i v \sigma d x+\int_{\Omega} f \cdot v d x=0 \forall v \in L_{d i s}^{2}(\Omega) .
\end{aligned}
$$

One can see that (12)-(13) practically coincide with the variational formulation of the Hellinger-Reissner principle. The use of this principle in the framework of finite elements can be traced Back to the pioneering work of Herrmann [9] and Hellan [10]. The interest in using the stress field $\sigma$ as an independent variable is questionable in as simple a case as the present one, but it is clear in more general and more complicated problems involving nonlinearities, plasticity, and so on.

Let the bilinear forms $\mathrm{a}$ and $\mathrm{b}$, and the linear forms 1 and s such that:

$$
\begin{gathered}
a(\sigma, \tau)=\int_{\Omega}(E: \sigma): \tau d x \\
b(\sigma, u)=\int_{\Omega} u \cdot \operatorname{div} \sigma d x \\
l(v)=-\int_{\Omega} f \cdot v d x
\end{gathered}
$$

$$
s(\tau)=\int_{\Gamma_{u}} \bar{u} \tau . n d \Gamma, \quad \text { for all } \tau \in H(\operatorname{div}, \Omega)
$$

The underlying weak formulation (12)-(13) may be restated as:

Find $\sigma \in H_{\bar{t}}$ and $u \in L_{d i s}^{2}(\Omega)$ such that:

$$
\begin{gathered}
a(\sigma, \tau)+b(\tau, u)=s(\tau), \text { for all } \tau \in H_{0}(\operatorname{div}, \Omega) \\
b(\sigma, v)=l(v), \text { for all } v \in L_{d i s}^{2}(\Omega) .
\end{gathered}
$$

THEOREM 1. Let $\mathrm{E}$ and $\Psi$ be real Hilbert spaces, $a\left(\xi_{1}, \xi_{1}\right)$ a bilinear form on $\mathrm{E} \times \mathrm{E}$, and $b(\xi, \psi)$ a bilinear form an $\mathrm{E} \times \Psi$. Set

$$
K=\{\xi \mid \xi \in E, b(\xi, \psi)=0 \forall \psi \in \Psi\},
$$

And assume that:

$\exists \alpha>0$, such that $a(\xi, \xi) \geq \alpha\|\xi\|_{E}^{2}, \forall \xi \in K$

$$
\begin{gathered}
\exists \beta>0, \text { such that } \underset{\xi \in E-\{0\}}{S U P} \frac{b(\xi, \psi)}{\|\xi\|_{E}} \geq \beta\|\xi\|_{\Psi}, \\
\forall \psi \in \Psi
\end{gathered}
$$

Then for every $l_{1} \in E^{\prime}$ and $l_{2} \in \Psi^{\prime}$ there exist a unique solution $(\bar{\xi}, \bar{\psi})$ of the problem

$$
\begin{gathered}
a(\bar{\xi}, \xi)+b(\xi, \bar{\psi})=\left\langle l_{1}, \xi\right\rangle, \text { for all } \xi \in E \\
b(\bar{\xi}, \psi)=\left\langle l_{2}, \psi\right\rangle, \text { for all } \psi \in \Psi .
\end{gathered}
$$

REMARK 1. If problem (21)-(22) has a unique solution for every $l_{1} \in E^{\prime}$ and $l_{2} \in E^{\prime}$, then (20) holds and the bilinear form $a\left(\xi_{1}, \xi_{2}\right)$ restricted to $\mathrm{K}$, is nonsingular (in the sense that it induces an isomorphism from $\mathrm{K}$ onto $\mathrm{K}$ '). Clearly if one assumes that a $\left(\xi_{1}, \xi_{2}\right)$ is symmetric and positive semi definite, then (21) and (22) are necessary and sufficient for the existence and uniqueness of the solution of (23)-(24).

REMARK 2. It is clear that if a $\left(\xi_{1}, \xi_{2}\right)$ is symmetric; the solution $(\bar{\xi}, \bar{\psi})$ of (23)-(24) minimizes the functional

$$
J(\xi)=\frac{1}{2} a(\xi, \xi)-\left\langle l_{1}, \xi\right\rangle
$$

On the subspace of $\mathrm{E}$,

$$
K\left(l_{2}\right)=\left\{\xi \mid \xi \in E, b(\xi, \psi)=\left\langle l_{2}, \psi\right\rangle \forall \psi \in \Psi\right\}
$$

And the formulation (23)-(24) corresponds to the introduction in (25)-(26) of the Lagrange multiplier $\bar{\xi}$.

\section{MIXED FINITE ELEMENT APPROXIMATION}

Let $T_{h} ; h>0$, be a family of rectangulations of $\Omega$.

The edges of elements will be denoted $e_{i}(i=1,2,3$ or $i=1,2,3,4)$ in the two-dimensional case. Let us deal first with the abstract framework (23)-(24). Assume that we are 
given two sequences $\left\{E_{h}\right\}_{\mathrm{h}>0}$ and $\left\{\Psi_{h}\right\}_{\mathrm{h}>0}$ of subspaces $\mathrm{E}$ and $\Psi$, respectively.

We set

$$
K_{h}=\left\{\xi_{h} \mid \xi_{h} \in E_{h}, b\left(\xi_{h}, \psi_{h}\right)=0 \forall \psi_{h} \in \Psi_{h}\right\} \text {. }
$$

We have the following approximation theorem

THEOREM 2. Assume that

$$
\begin{gathered}
\exists \alpha_{h}>0 \text {, such that } a(\xi, \xi) \geq \alpha_{h}\|\xi\|_{E}^{2}, \forall \xi \in K_{h} \\
\exists \beta_{h}>0 \text {, such that } \underset{\xi \in E_{h}-\{0\}}{S U P} \frac{b(\xi, \psi)}{\|\xi\|_{E}} \geq \beta_{h}\|\psi\|_{\Psi} \\
\forall \psi \in \Psi_{\mathrm{h}}
\end{gathered}
$$

Then for every $l_{1} \in \mathrm{E}^{\prime}$ and $l_{2} \in \Psi^{\prime}$, and for every $h>0$, the discrete problem

$$
\begin{gathered}
a\left(\bar{\xi}_{h}, \xi\right)+b\left(\xi, \bar{\psi}_{h}\right)=\left\langle l_{1}, \xi\right\rangle, \text { for all } \xi \in E_{h}, \\
b\left(\bar{\xi}_{h}, \psi\right)=\left\langle l_{2}, \psi\right\rangle \text {, for all } \psi \in \Psi_{h}
\end{gathered}
$$

Has a unique solution. Moreover, there exists a constant $\gamma_{h}\left(\alpha_{h}, \beta_{h}\right)>0$ such that

$$
\begin{aligned}
\left\|\bar{\xi}-\bar{\xi}_{h}\right\|_{E}+\left\|\bar{\psi}-\bar{\psi}_{h}\right\|_{\Psi} & \leq \gamma_{h}\left(_{\xi_{h} \in E_{h}} \inf \left\|\bar{\xi}-\xi_{h}\right\|_{E}\right. \\
& \left.+\underset{\psi_{h} \in \Psi_{h}}{\inf }\left\|\bar{\psi}-\psi_{h}\right\|_{\Psi}\right) .
\end{aligned}
$$

The dependence of $\gamma_{h}$ on $\alpha_{h}$ and $\beta_{h}$ can be easy traced [8]. Clearly if (21) and (22) hold with constants $\bar{\alpha}$ and $\bar{\beta}$ independent of $\mathrm{h}$, then (32) holds with a constant $\bar{\gamma}$ independent of $h$.

We define in general, for $\mathrm{m}$ integer $\geq 0$,

$$
H^{m}(\Omega)=\left\{v\left|D^{\alpha} v \in L^{2}(\Omega), \forall\right| \alpha \mid \leq m\right\}
$$

Where

$$
D^{\alpha} v=\frac{\partial^{|\alpha|} v}{\partial x_{1}^{\alpha_{1}} \ldots \partial x_{n}^{\alpha_{n}}},|\alpha|=\alpha_{1}+\ldots+\alpha_{n}
$$

These derivatives being taken in the sense of distributions. On this space, we shall use the semi-norm

$$
|v|_{m, \Omega}^{2}=\sum_{|\alpha|=m}\left|D^{\alpha} v\right|_{L^{2}(\Omega)}^{2}
$$

and the norm

$$
\|v\|_{m}^{2}=\sum_{K \leq m}|v|_{k, \Omega}^{2}
$$

We are now ready for the error estimates.

THEOREM 3. If $(\sigma, u)$ is the solution of (12)-(13) and $\left(\sigma_{h}, u_{h}\right)$ is the solution of (30)-(31), there exist a constant C $>0$ such that:

$\left\|\sigma-\sigma_{h}\right\|_{0}+\left\|u-u_{h}\right\|_{0} \leq C h^{2}\left(\|\sigma\|_{2}+\|u\|_{3}\right)$.
Discretization of the mixed formulations, for linear elliptic operators, many examples of successful discretization of (12)-(13) are known. The first ones were introduced by Raviart and Thomas in [11] and then reelaborated and extended to more general cases by Nedelec [12]. Other families of possible discretization were introduced years later by Brezzi, Douglas, and Marini [1][13].

To give a more precise definition of our mixed finite element approximation we shall need a few definitions. Let us define on an element $\mathrm{K}$.

$\mathrm{P}_{\mathrm{k}}$ : the space of polynomials of degree $\leq \mathrm{k}$.

We shall also need polynomial spaces on the edges of the elements

$$
\begin{aligned}
& R_{k}(\partial K)=\left\{\phi\left|\phi \in L^{2}(\partial K), \phi\right|_{e_{i}} \in P_{k}\left(e_{i}\right),\right. \\
& \left.\forall e_{i} \in \partial K\right\} .
\end{aligned}
$$

In the two-dimensional, for the triangular elements we have

$$
\begin{gathered}
B D M_{k}(K)=\left(P_{k}(K)\right)^{2},(k \geq 1) \\
B D F M_{k}(K)=\left\{\begin{array}{c}
q \in\left(P_{k}(K)\right)^{2}|q \cdot n|_{\partial K} \\
\left.\in R_{k-1}(\partial K)\right\},(k \geq 1)
\end{array}\right. \\
R T_{k}(K)=\left(P_{k}(K)\right)^{2} \oplus x\left(P_{k}(K)\right),(k \geq 1) .
\end{gathered}
$$

Restricting $q \in B D M_{k}(K)$ to have a normal trace in $R_{k-1}(\partial K)$ yields a space larger than $R T_{k}(K)$, but having essentially the same properties, that we denote $B D F M_{k}(K)$.

The dimension of $B D M_{k}$ is thus

$$
\operatorname{dim} B D M_{k}= \begin{cases}(k+1)(k+2) & \text { for } n=2 \\ \frac{1}{2}(k+1)(k+2)(k+3) & \text { for } n=3 .\end{cases}
$$

For the triangular case we thus have the following inclusions between the spaces just defined

$$
\begin{aligned}
R T_{0} \subset B D F M_{1} & \subset B D M_{1} \subset R T_{1} \subset B D F M_{2} \subset B D M_{2} \\
& \subset R T_{2}
\end{aligned}
$$

We consider the space obtained basically from the space of Brezzi-Douglas-Marini.

$$
B D M_{1}(K)=\left(P_{1}(K)\right)^{2} .
$$

We have

$$
\begin{gathered}
\operatorname{div}\left(B D M_{1}(K)\right)=P_{0}(K) . \\
\operatorname{dim} B D M_{1}=6
\end{gathered}
$$



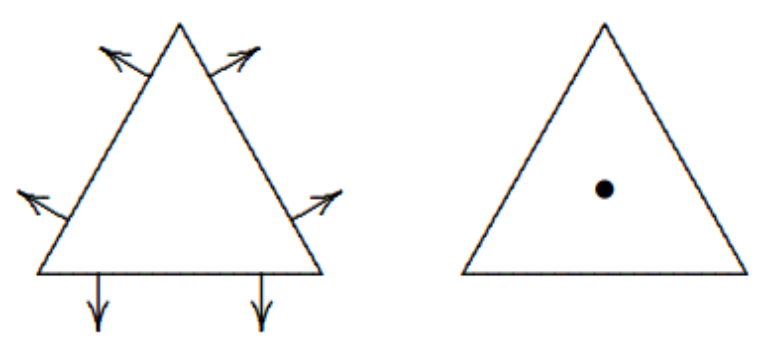

Fig. 2. . Brezzi - Douglas - Marini element, the element diagrams for the stress and displacement elements.

The discrete scheme allows the utilization of $\mathrm{BDM}_{1}$ for the stress tensor and piecewise constant elements for the displacement.

We define, for choice of $\mathrm{BDM}_{1}(\mathrm{~K})$, a space

$$
\begin{gathered}
W_{h}=\left\{\tau_{h} \in H(\operatorname{div}, \Omega),\left.\tau_{h}\right|_{K} \in B D M_{1}(K)\right. \\
\left.\forall K \in T_{h}\right\}
\end{gathered}
$$

$$
V_{h}=\left\{v_{h} \in L_{\text {dis }}^{2}(\Omega),\left.v_{h}\right|_{K} \in P_{0}(K) \forall K \in T_{h}\right\} .
$$

We chose finite dimensional subspace $H_{0}^{h}(\operatorname{div}, \Omega) \subset$ $H_{0}(\operatorname{div}, \Omega)$.

A mixed finite element approximation of (12)-(13) is defined by

Find $\sigma_{h} \in \mathrm{W}_{\mathrm{h}}$ and $u_{h} \in V_{h}$ such that

$$
\begin{gathered}
\int_{\Omega}\left(E: \sigma_{h}\right): \tau_{h} d x+\int_{\Omega} u_{h} \cdot \operatorname{div} \tau_{h} d x=\int_{\Gamma_{u}} \bar{u} \tau_{h} \cdot n d \Gamma \\
\forall \tau_{h} \in H_{0}^{h}(\operatorname{div}, \Omega) \\
\int_{\Omega} v_{h} \cdot d i v \sigma_{h} d x+\int_{\Omega} f \cdot v_{h} d x=0 \forall v_{h} \in V_{h} .
\end{gathered}
$$

We obtain a system of linear equations

$$
\left(\begin{array}{cc}
A & B^{T} \\
B & 0
\end{array}\right)\left(\begin{array}{l}
T \\
U
\end{array}\right)=\left(\begin{array}{l}
S \\
L
\end{array}\right) \text {. }
$$

Where $S=\left[s_{m}\right], L=\left[l_{m}\right]$.

The matrix associated for the system (51) is symmetric indefinite. We use the iterative methods Minimum Residual Method (MINRES) for solving the symmetric system.

\section{NUMERICAL SIMULATIONS}

\section{Example 1. Circular Void in a Finite Plate}

Here a void of radius 0.3 is placed in the center of a plate of size $3 \times 3$ which is subjected to a unit stress in the y-direction.

The stress plot for $\sigma_{y y} \mathrm{~s}$ is in excellent agreement with the expected $3 \sigma$ stress concentration at the edges of the hole.

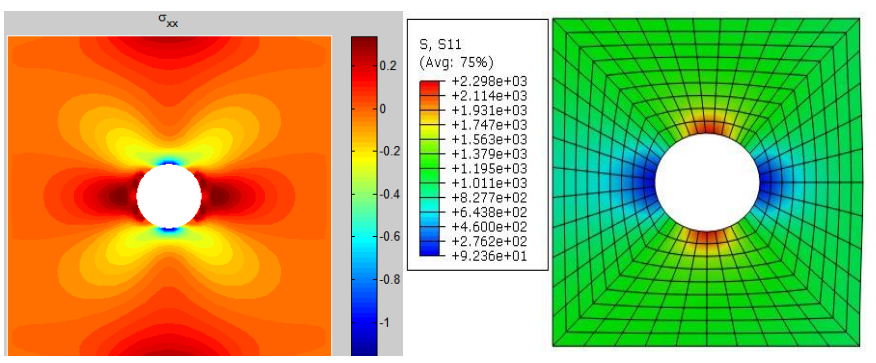

Fig. 3. Stress Solution $\Sigma_{x x}$ By Mixed Finite Element Method (Left) And Stress Solution (Right) Computed By ABAQUS.

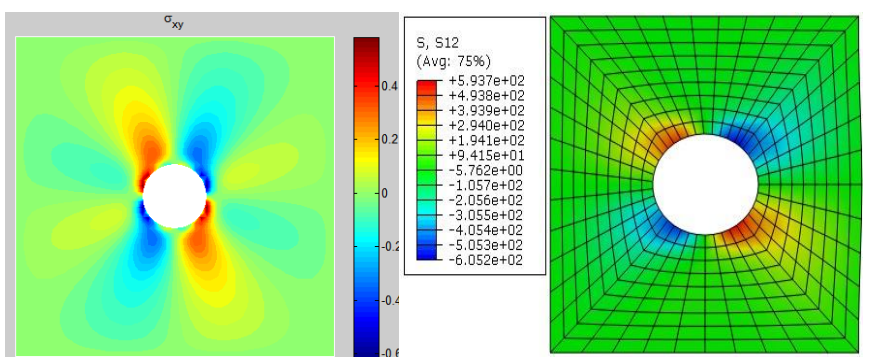

Fig. 4. Stress $\Sigma x y$ By Mixed Finite Element Method (Left) And Stress Solution (Right) Computed By ABAQUS.

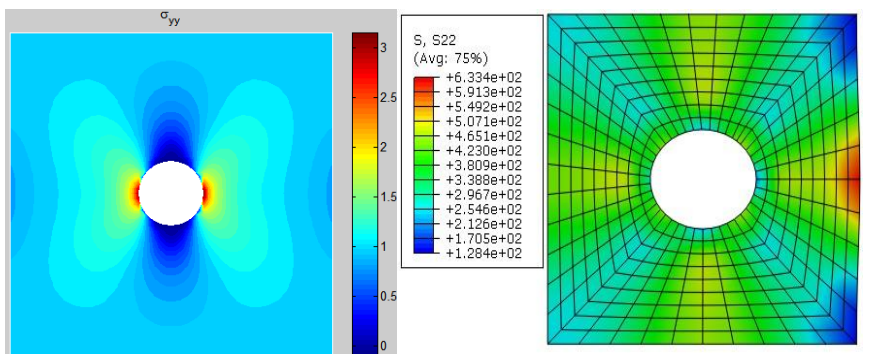

Fig. 5. Stress $\Sigma_{\text {yy }}$ By Mixed Finite Element Method (Left) And Stress Solution (Right) Computed By ABAQUS.
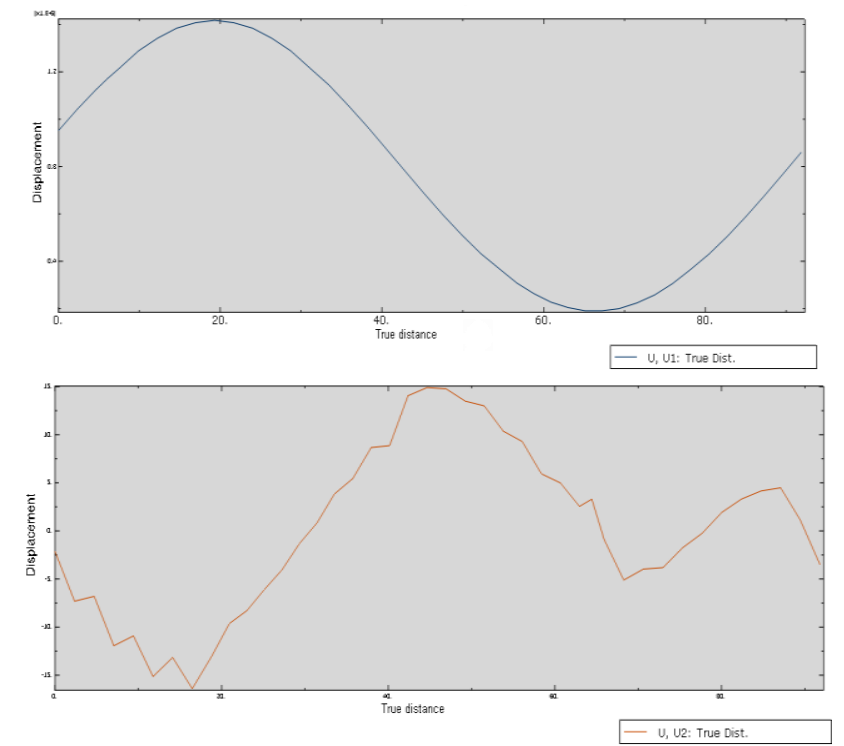

Fig. 6. Curve Of The Displacement Ux And Uy Along Hole In A Finite Plate 
Example 2. Circular Inclusion in a Finite Plate

Here an inclusion with $\mathrm{E}=70 \mathrm{gpa}$ and $v=0.3$ and radius 0.5 is placed in the center of a plate of size $6 \times 10$ with $\mathrm{E}=50 \mathrm{gpa}$ and $v=0.3$ which is subjected to a unit tension in the $y$-direction.

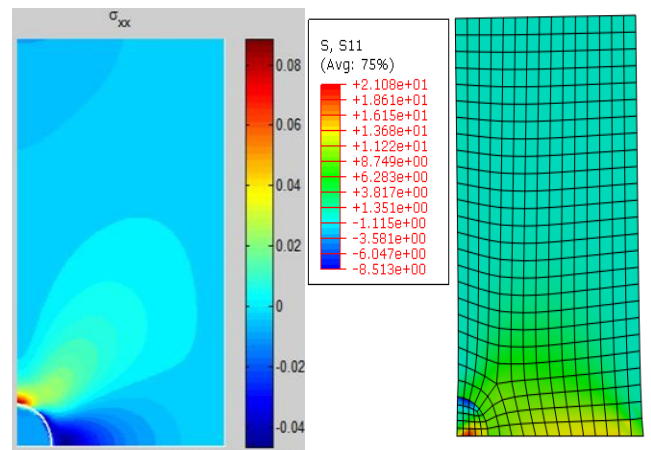

Fig. 7. Stress $\Sigma_{x x}$ By Mixed Finite Element Method (Left) And Stress Solution (Right) Computed By ABAQUS.

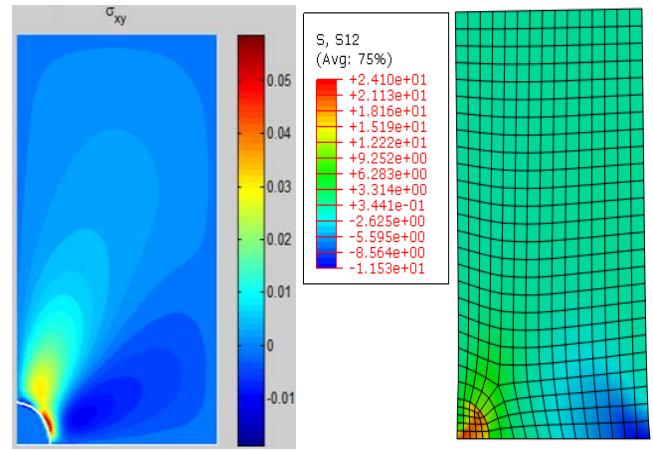

Fig. 8. Stress $\Sigma_{\mathrm{xy}}$ By Mixed Finite Element Method (Left) And Stress Solution (Right) Computed By ABAQUS.

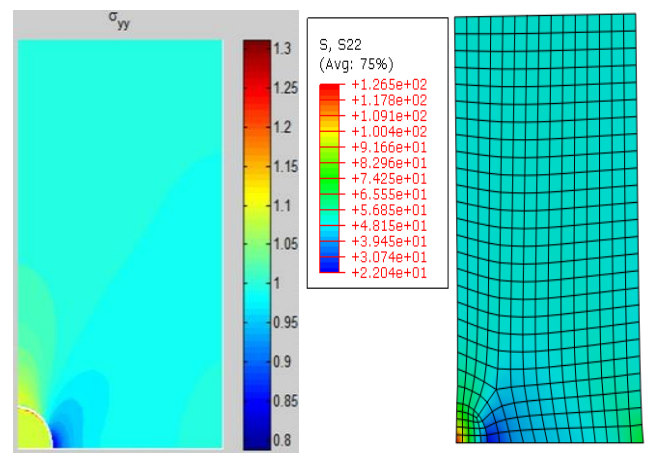

Fig. 9. Stress $\Sigma$ yy By Mixed Finite Element Method (Left) And Stress Solution (Right) Computed By ABAQUS.
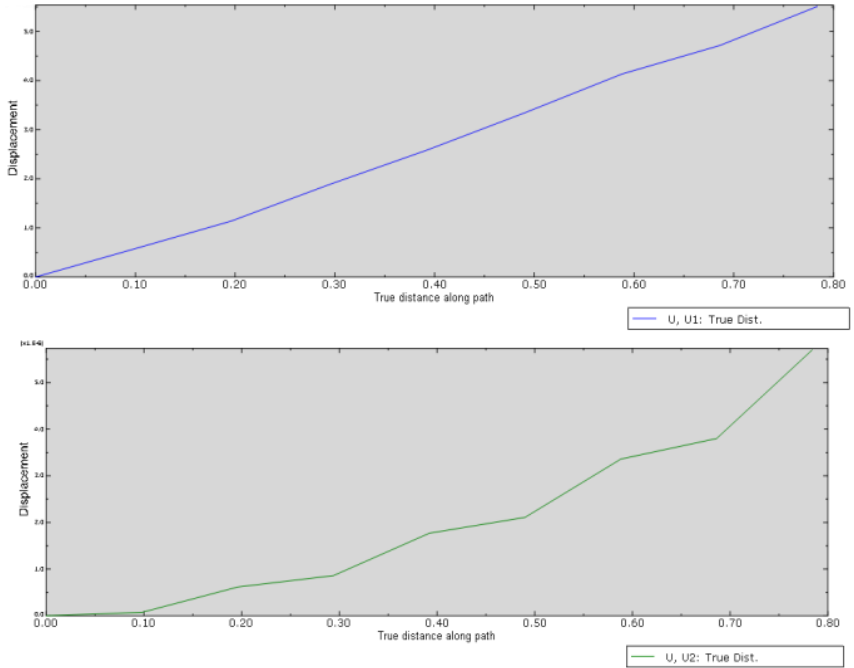

Fig. 10. Curve Of Displacement Ux And Curve Of Displacement Uy Along Inclusion In A Finite Plate.

\section{CONCLUSION}

We were interested in this work in the numeric solution for equilibrium equations. It includes algorithms for discretization by mixed finite element methods. The discrete scheme allows the utilization of $B D M_{1}$ for the stress tensor and piecewise constant elements for the displacement. Our results agree with ABAQUS.

Numerical results are presented to see the performance of the method, and seem to be interesting by comparing them with other recent results.

\section{ACKNOWLEDGMENTS}

The authors would like to express their sincere thanks for the referee for his/her helpful suggestions.

\section{REFERENCES}

[1] F. Brezzi, J. Douglas, M. Fortin, L. Marini, Efficient rectangular mixed finite elements in two and three variables, RAIRO Model. Math. Anal. Number. vol. 21, issue. 3, pp. 581-604, 1987.

[2] W. Qiu and L. Demkowicz, Mixed hp-finite element method for linear elasticity with weakly imposed symmetry: stability analysis, SIAM J. Numer. Anal., vol. 49, n o. 2, pp. 619-641, 2011.

[3] J. Guzman, A unified analysis of several mixed methods for elasticity with weak stress symmetry, J. Sci. Comput., vol. 44, pp. 156-169, 2010.

[4] D.N. Arnold, J. Douglas Jr., C.P. Gupta, A family of higher order mixed finite element methods for plane elasticity, Numer. Math., vol. 45, pp. 1-22, 1984.

[5] C. Johnson and B. Mercier, Some equilibrium finite element methods for two-dimensional elasticity problems, Numer. Math., vol. 30, pp. 103-116, 1978.

[6] F. Brezzi, M. Fortin. Mixed and Hybrid Finite Element Method. Springer Verlag; New York, 1991.

[7] Douglas N. Arnold, Jim Douglas, Jr., and Chaitan P. Gupta. A Family of Higher Order Mixed Finite Element Methods for Plane Elasticity. Number. Math., vol. 45, pp. 1-22, 1984.

[8] F. Brezzi, On the existence uniqueness and approximation of saddle point problems arising from Lagrangian multipliers, RAIRO, vol. 8-32, pp. 129$151,1974$.

[9] L.R. Herrmann, Finite element bending analysis for plates, J. Eng. Mech. Div. ASCEEMS, vol. 93, pp. 49-83, 1967. 
[10] K. Hellan, Analysis of elastic plates in flexure by a simplified finite element method, Acta Polytech. Scand. Math. Comput. Sci. Ser., vol. 46, 1967.

[11] P.A. Raviart and J.M. Thomas, A mixed finite element method for 2nd order elliptic problems, Mathematical Aspects of Finite Element Methods (Proc. Conf., Consiglio Naz. delle Richerche, Rome, 1975), Lecture Notes in Math., vol. 606, Springer-Berlag, New York, pp. 292-315, 1977.
[12] J.C. Nedelec, Mixed finite elements in R3, Numer. Math., vol. 35, pp. 315$341,1980$.

[13] F. Brezzi, J. Douglas, Jr., and L.D. Marini, Two families of mixed finite elements for second order elliptic problems, Numer. Math., vol. 47, pp. 217-235, 1985.

[14] I. Babuska, The finite element method with lagrangian multipliers, Numer. Math., vol. 20, pp. 179-192, 1973.

[15] M. Crouzeix and P.A. Raviart, Conforming and non-conforming finite element methods for solving the stationary Stokes equations, R.A.I.R.O. vol. 7, pp. 33-76, 1973.

[16] R.S. Falk and J. Osborn, Error estimates for mixed methods,R.A.I.R.O. vol.4, pp. 249-277, 1980.

[17] M. Fortin, An analysis of the convergence of mixed finite element methods, R.A.I.R.O. vol.11, pp. 341-354, 1977.

[18] J.E. Roberts and J. M. Thomas, Mixed and Hybrid Methods in Handbook of Numerical Analysis, Vol. II (P.G. Ciarlet and J.L. Lions, eds.), Finite Element Methods (Part 1),North Holland, 1989.

[19] V. Girault and P.A. Raviart, Element Methods for Navier-Stokes Equations, Springer, Berlin Heidelberg New York, 1986. 